\title{
Basrah Journal
} Of Surgery

Bas J Surg, December, 24, 2018

\section{BLEEDING FOLLOWING BARIATRIC SURGERY (TYPES \& MANAGEMENT MODALITIES)}

\author{
Falih Mohsen Al-Gazgooz ${ }^{*}$ \& Abdulkareem Jabbar Ghadban Al- \\ Ebadi $^{@}$ \\ *,@ MB,ChB, CABS, FACS Consultants in Laparoscopic \& Bariatric Surgery, Al-Sadr Teaching Hospital, \\ Basrah, Iraq.
}

\begin{abstract}
Bleeding is a serious complication following bariatric procedures such as Minigastric bypass (MGB), Roux-En-Y Gastric Bypass (RYGB) Surgery, and Sleeve Gastrectomy (SG). Post bariatric bleeding is either acute or chronic. Although this complication is declined now, but still it is important to know because if it is not managed urgently it will lead to serious results. The source of bleeding may be from the staple lines, unexpected injury to the spleen, or to one of the blood vessels around the surgical field.

The other source of bleeding may be internal through the GIT and it is presented as hematemesis or melena. Gastrointestinal bleeding in patients underwent bariatric surgery may occur in the esophagus, gastric pouch, new sleeved stomach, the Roux limb just distal to the anastomosis, the remaining of the small intestine. The site of bleeding can be determined and treated mostly by endoscopy but in certain cases may require re-laparoscopy.

The aim of this study is to analyze the incidence, types and management of this bleeding.

This is a prospective study which has been done in Al-Sadr Teaching Hospital, Basrah, Iraq, from June 2015 to June 2017. The study included 540 case who were subjected to SG, RYGB, MGB and they were analyzed regarding; time of occurrence, postoperative bleeding, types whether intra-peritoneal or intra-luminal and type of approaches for treating this bleeding. In our study we don't over sewn the staple line and didn't use any buttressing material or synthetic coagulation powder.

The total number of patients developed post-operative bleeding were 18 cases out of 540,12 after sleeve gastrectomy, 3 after mini gastric bypass and 3 after Roux-En-Y. Regarding sleeve gastrectomy; two patients of the required re-laparoscopy and evacuation of hematoma with treating site of bleeding by clipping or suturing according to the condition, three patients required aspiration under ultrasound guide, while the remaining 7 cases were kept only under conservative measures. The 3 patient of the MGB were managed conservatively and the 3 cases of Roux-En-Y were managed either conservatively in 2 cases or by endoscopic intervention in one case.

In conclusion, we advise the surgeons to use reinforcement of staple line to decrease incidence of post-operative bleeding.
\end{abstract}

\section{Introduction}

besity is a major health problem worldwide that leads to increased incidence of morbidity and mortality. People are generally regarded obese when their body mass index (BMI) exceeded $30 \mathrm{~kg} / \mathrm{m}^{2}$, and above the $25 \mathrm{~kg} / \mathrm{m}^{2}$ were regarded as overweight ${ }^{1}$.

Obesity is a risk factor of many diseases like cardiovascular diseases, diabetes, sleep apnea, osteoarthritis, depression, and rarely cancer $^{2}$. Life style modification such as change in diet quality and exercise can control obesity. If diet modification, exercise, and medication failed to control morbid obesity, a gastric balloon or surgery should be considered to solve the problem. The choice of procedure depends on BMI, food habit, co-morbid diseases and psychological state $^{3}$.

Bariatric surgery is regarded as the best modality that gives a significant weight loss for morbidly obese patients and according to a medical guideline by 
the American College of Physicians, bariatric surgery is indicated as a treatment for patients with a BMI of $40 \mathrm{~kg} / \mathrm{m}^{2}$ and above or patients with BMI $35-40 \mathrm{~kg} / \mathrm{m}^{2}$ with co-morbid diseases such as diabetes mellitus, hypertension, sleep apnea etc. After failure of conservative measure for control obesity, the surgeon should explain the type of procedure that the patient may need possible early complications like bleeding and leak, possible re- exploration and long term complications ${ }^{4}$.

Also certain centers included patients with BMI of 30 to $34.9 \mathrm{~kg} / \mathrm{m}^{2}$ with diabetes or other metabolic disorders as indication of bariatric surgery, but there is controversy regarding benefits in those patients, this is determined by the International Diabetes Federation issued in 2011 .

Psychiatric assessment in addition to endocrine, pulmonary, and cardiac assessment are vital for patients considered for surgery especially in those with co-morbid diseases. Surgical patients are candidates for depression especially those with a BMI of $40 \mathrm{~kg} / \mathrm{m}^{2}$ and above which have 5 fold depression risk ${ }^{6}$.

Although there are no absolute contraindications for bariatric surgery, but there are some relative contraindications such as severe heart failure, unstable coronary artery disease, end-stage lung disease, active cancer treatment, portal hypertension, and drug or alcohol dependency. In Roux-En-Y Gastric Bypass (RYGB), Crohn's disease is regarded a relative contraindication in addition to any contraindication for general anesthesia ${ }^{7}$.

Bleeding, as a postoperative complication, may be revealed through surgical drain, hematemesis or melena with or without tachycardia, hypotension and oliguria ${ }^{8}$. Nowadays, bleeding is regarded as uncommon complication with incidence of about $0-4.4 \%$ depending on the type of surgery whether they use new hemostatic agents like fibrin glue, in addition to reinforcement techniques such as suturing or buttressing agents ${ }^{9}$.

Many factors can cause bleeding including lengthy staple line especially in sleeve gastrectomy and the change in intra-gastric pressure. Prophylactic heparinization with low molecular weight heparins is an another cause for postoperative bleeding. Postsurgical bleeding is either intra-luminal bleeding (ILB) or intra-abdominal bleeding (IAB). Intra-luminal bleeding occurs usually from the staple line and presented in the form of hematemesis and melena with or without tachycardia and hypotension which is mostly managed conservatively ${ }^{10}$.

On the other hand cases with intraabdominal bleeding presented early postoperatively with gradual decrease in hemoglobin level associated with decrease in blood pressure and increase in pulse rate. Usual sites for intra-abdominal bleeding include; the staple line, spleen, liver or abdominal wall near sites of trocar entry $^{11}$.

As result of that, hematoma or abscess may occur apart from bleeding from drain or nasogastric tube which is regarded as risky sign and needs urgent management with serial checking of hemoglobin and bleeding profile ${ }^{12}$. Many authors prefer reinforcement over sewing the staple line to reduce postoperative bleeding, although this elongate the time of surgery with risk of narrowing lumens ${ }^{13}$. Other hemostatic agents, such as fibrin glue put on staple anastomotic line are very helpful for hemostasis and declining postoperative bleeding $^{14}$.

Other complications include: Leak from site of staple line in gastric sleeve or from anastomosis site in bypass procedure. Thrombotic complications may occur in spite of prophylactic measures. Cardiac and pulmonary complications in addition to iatrogenic injuries may also occur.

This study aimed to analyze the incidence, types and modalities of 
management of post bariatric surgery bleeding.

\section{Patients and Methods}

This prospective study was done on 540 patients with morbid obesity BMI from $38-52 \mathrm{~kg} / \mathrm{m}$ with age range from 18-63 year in Al-Sadr Teaching Hospital, Basrah, Iraq in the period from June 2015 to June 2017. All patients were thoroughly assessed preoperatively by laboratory investigations including complete blood picture, liver function test, renal function test, blood sugar analysis, bleeding profile (all cases of disturbed coagulation profile are excluded), Hormones assay like serum cortisone level, thyroid function test. Cardiac assessment is also necessary and some patients may need psychological assessment.

All measures of preventing thromboembolic complications were done perioperatively starting from preoperative low molecular weight heparin, use of stocking intraoperatively and continuing heparin for 10-15 days postoperatively.

Some of patients required oesophageogastro-doudenoscopy (OGD) according to the American Society for Metabolic and bariatric Surgery guide lines. Patients were categorized into three groups: Sleeve gastrectomy (SG) 388 cases (71.9\%), Minigastric bypass (MGB) 117 cases (21.6\%), and Roux-En-Y Gastric Bypass (RYGB) 35 cases (6.5\%). The frequency, types, and management modalities were analyzed according to time and type of bleeding.

In this study, we did not use any buttressing material or glue and did not reinforce the staple line by suture. Two types of staplers Covedien and Eschelone which are powered or mechanical were used. Nasogastric bougie 36 french was used as a guide and to determine the size of gastric pouch. In SG, we complete the operation by 5-7 staplers with no oversewing but we used clip for any bleeding point. Five ports were inserted inside the abdomen and $\mathrm{CO}_{2}$ insufflation was started. The liver was retracted and division of greater omentum 5-6 cm from pylorus till left crus of diaphragm was done. After insertion of bougie tube, stapling was started by thick 4-5 mm stapler, then the stapler size was decreased for the next stapling preserving good size of antrum and take care not narrowing the incisura. Once complete division of stomach was accomplished, the gastric remnant was taken out, drain was inserted and left intra-abdominally for 24 hour unless there are any complication, the nasogastric tube was removed. In MGB, trocar position is variable according to surgeon preference, 5 trochers were used. The pouch length was considered to be about 15-18 cm. Then bypass was performed about $180 \mathrm{~cm}$ from the duodeno-jejunal flexture by creating gastro-jejunostomy with the pouch. Staplers were utilized in creating the pouch and posterior anastomosis between the pouch and jejunum while the anterior anastomosis was done by handsoversewing. In RYGB, a small pouch was created of size $20-30 \mathrm{~cm}$ from the upper portion of stomach by staplers connecting the pouch to roux limb of about $150 \mathrm{~cm}$ (alimentary limb) then anastomozed with bilo-pancreatic limb (which is of $50 \mathrm{~cm}$ length from duodeno-jejunal flexture.

Most of the patients were discharged home after 48 hours, unless they have any complication. The patients were instructed about postoperative nutrition and we follow the patients for at least three years according to the type of surgery and complications.

Data was analyzed statistically using SPSS program and a $\mathrm{P}$ value of less than 0.05 was considered as statistically significant.

\section{Results}

The patients' demographic characteristics are demonstrated in Table I, showing that mean age was 44.6 , the male gender was 178 and the BMI mean was 41.5. 
Table I: Demography of the patients

\begin{tabular}{|l|l||}
\hline Patients characteristics & Number (\%) \\
\hline Age mean (range) & $44.6 \quad(18-63)$ years \\
\hline Gender & $178(32.9 \%)$ males \\
\hline BMI ( kg/m2) & $41.5(38-52)$ \\
\hline Diabetes & $152(28.1 \%)$ \\
\hline Hypertension & $96(17.8)$ \\
\hline Ischemic heart disease & $24(4.5)$ \\
\hline Bleeding disorders & $0.0(0.0 \%)$ \\
\hline Use of anticoagulants & $0.0(0.0 \%)$ \\
\hline $\begin{array}{l}\text { Type of surgery: } \\
\text { Sleeve gastrectomy } \\
\text { Mini gastric bypass } \\
\text { Roux-en-Y gastric bypass }\end{array}$ & $388(71.9 \%)$ \\
\hline
\end{tabular}

The total number of postoperative bleeding was 18 patients (3.4\%), 12 (2.3\%) of them after SG which were all intra-peritoneally, $3(0.6 \%)$ after RYGB and were intra-luminal, and $3(0.6 \%)$ patients after MGB, one intra-luminal bleeding and two intra-peritoneal. From those patients after SG, two patients required re-laparoscopy and evacuation of hematoma with treating the site of bleeding by clipping or suturing according to the condition, three patients required aspiration under ultrasound guide while the remaining cases were kept only under observation and conservative measures. In three cases after RYGB, only one case required endoscopic clip and injection therapy while other two cases were kept on conservative measures. In MGB, all the three cases of the bleeding were treated conservatively as shown in Table II.

Table II: Frequency of postoperative bleeding according to type of surgery \& modality of treatment

\begin{tabular}{|l|c|c|c|c|c|}
\hline \multirow{2}{*}{$\begin{array}{l}\text { Type of } \\
\text { surgery }\end{array}$} & \multirow{2}{*}{ Bleeding } & \multicolumn{3}{|c|}{ Management modality number \& percentage } \\
\cline { 3 - 6 } & No. (\%) & No. (\%) & $\begin{array}{l}\text { Enservative } \\
\text { intervention } \\
\text { No. (\%) }\end{array}$ & Nelaparoscopy & Aspiration \\
& $12(2.3 \%)$ & $7.0(58.4 \%)$ & $0.0(0.0 \%)$ & $2(16.6 \%)$ & No. (\%) \\
\hline $\begin{array}{l}\text { Sleeve } \\
\text { gastrectomy }\end{array}$ & $3(0.6 \%)$ & $3(100.0 \%)$ & & & \\
\hline $\begin{array}{l}\text { Mini gastric } \\
\text { bypass }\end{array}$ & $3(0.6)$ & $2(66.6 \%)$ & $1(33.4 \%)$ & & \\
\hline $\begin{array}{l}\text { Roux-en-Y } \\
\text { GB }\end{array}$ & $3(2.4 \%)$ & $12(66.7 \%)$ & $1(5.5 \%)$ & $2(11.2 \%)$ & $3(16.6 \%)$ \\
\hline Total & $18(3.4 \%)$ & & & \\
\hline
\end{tabular}

The frequency of bleeding in relation to the type of surgery was shown in Table III demonstrating that Roux-En-Y procedure carry the highest percentage of postoperative bleeding (8.6\%) and the mini bypass is lowest. The $\mathrm{p}$ value was $>0.05$ which denotes non-significant results so there was no difference between the types of surgery regarding postoperative bleeding. 
Table III: Frequency of postoperative bleeding in relation to type of surgery

\begin{tabular}{|l|c|c|c|}
\hline \multicolumn{1}{|c|}{$\begin{array}{c}\text { Type of } \\
\text { surgery }\end{array}$} & $\begin{array}{c}\text { Patients without } \\
\text { postoperative bleeding }\end{array}$ & $\begin{array}{c}\text { Patients with postoperative } \\
\text { bleeding }\end{array}$ & Total \\
\hline $\begin{array}{l}\text { Sleeve } \\
\text { gastrectomy }\end{array}$ & 376 & $12(3.09 \%)$ & 388 \\
\hline $\begin{array}{l}\text { Mini gastric } \\
\text { bypass }\end{array}$ & 114 & $3(2.6 \%)$ & 117 \\
\hline Roux-en-Y GB & 32 & $3(8.6 \%)$ & 35 \\
\hline Total & 522 & $18(3.4 \%)$ & 540 \\
\hline
\end{tabular}

\section{Discussion}

The gastro-intestinal bleeding is the presence of hematemesis or melena or continuous bleeding from surgical drain after surgery. This study concentrated on post bariatric surgery bleeding which can be intra-luminal or intra-peritoneal and can occur at any site of gastrointestinal tract. Bleeding can be classified according to the onset into acute which may occur in the first week, early in the 1-6 week, late in the 6-12 week, and chronic occur after 12 week $^{15}$. Usually the intra-peritoneal bleeding occurs as acute postoperative bleeding and mostly in staple line or near trocar site and vascular beds. Other causes of intra-abdominal bleeding is incomplete hemostasis at sites of dissection while intra-luminal bleeding may occur at any time due to surgical or medical causes like peptic ulcer or tumors. Bleeding in sleeve gastrectomy is mostly due to long staple line or short gastric vessel remnants or may be from trocar sites, while gastric bypass bleeding is resulted from stress ulcer or may be due to staple line ${ }^{16}$. Late and chronic bleeding usually occurs 6 weeks after surgery which is mostly due to marginal ulcer, gastritis or malignancy. Gastric bypass is the most common procedure in which chronic bleeding may occur because of ulceration at gastrojejunostomy site or some time occur in duodenum or gastric remnant which may be due to tension, ischemia, or NSAID ${ }^{17}$.

It is important to diagnose bleeding as early as possible, usually we put drain for 24 hours so acute bleeding can be detected through the drain. Other signs included abdominal distension, tachycardia, hypotension and oliguria. The most sensitive sign is tachycardia so patients usually kept on close observation especially in first 24 hours to observe any early sign of bleeding or other complications. When no sign or symptoms appear, the drain is our guide for early bleeding; the pattern of tachycardia in bleeding is cyclical ${ }^{18}$.

In this study, we follow the patients for 24-48 hours inside the hospital for any acute complication, 18 patients developed bleeding (3.4\%), 12 of them after sleeve gastrectomy (3.09\%), 3 after RYGB (8.6\%), and 3 after MGB (2.6\%).

This result does not differ from other centers that do not use reinforcement. Staple-line reinforcement (SLR) has important role in decreasing incidence of bleeding, SLR Includes many types like buttressing with absorbable materials such as glycolide, porcine small intestine submucosa, and recently the use of hemostatic agents such as floseal which also plays a good role in decreasing bleeding $^{19}$. Shikora et al. describe reinforcement in 2004; they follow buttressing material to reduce hemorrhage and leakage after GBP. They concluded that SLR with bovine pericardial strips has a role in decreasing acute staple-line failures ${ }^{20}$.

Hemostatic agents in GBP patients were discussed by Silecchia et al. to prevent leaks and bleeding, they have an important role in decreasing postoperative bleeding $^{21}$.

Another randomized trial from Dapriet al. found buttressing decreases hemorrhage; 
but no difference regarding other postoperative complications ${ }^{22}$. In 2015, Shikora published a meta-analysis to compare different types of SLR options, (no reinforcement group, over-sewing, bovine pericardial strips, and last group absorbable polymer membrane), the study compared bleeding rates as follow: $1.16 \%$ and 2.09\% with reinforcement (bovine pericardial strips and absorbable polymer membrane, respectively) in comparison with $4.94 \%$ and $2.41 \%$ for patients with no-reinforcement technique and the over suture, respectively it is clear from this analysis that reinforcement decreases incidence of bleeding ${ }^{23}$.

We found a difference in percentage of bleeding between the 3 procedures, but the difference is not statistically significant.

Apart from clinical assessment upper endoscopy and CT scan which is useful in stable patient to determine any collection or hematoma also useful in detecting any fluid in gastric remnant which raise suspicions of intra-luminal bleeding some time we need contrast to detect active bleeding.

Upper endoscopy is very useful diagnostic and therapeutic method to stop bleeding but must be used carefully in early postoperative period because it may disrupt anastomotic line ${ }^{24}$. If we don't find bleeding by endoscopy we should think of bleeding from other sites like jejunojejunostomy or gastric remnant, in such conditions we may need relaparoscopy or new modalities like capsule endoscopy to identify the site of bleeding ${ }^{25}$. When we suspect bleeding, we should admit the patient to the hospital for observation with good resuscitation. If the patient is stable, OGD should be done but if not stable and not respond to resuscitation, surgical intervention is a must. Most patients respond to conservative measures with no need for further intervention ${ }^{26}$. It is important to differentiate from the start whether the bleeding is intra-luminal or intra-abdominal because of difference in the management. Inra-abdominal bleeding is mostly treated conservatively but if no response, diagnostic laparoscopy should be done to localize the site of bleeding and deal with $\mathrm{it}^{27}$. In case of intra-luminal bleeding there is a role for conservative management but if no response, endoscopic intervention can be done to treat bleeding in gastric pouch in GBP by injection or cauterization ${ }^{28}$. In case of unstable patient, early re-exploration should be considered. If expert team is available, laparoscopy should be done before conversion to laparotomy when needed. Re-explorative laparotomy with or without intraoperative endoscopy is still regarded as effective diagnostic and therapeutic choice in those patients who have failed in conservative management, some authors regarded an emergency laparotomy as a safe choice ${ }^{28}$. Laparoscopically-assisted enteroscopy, is another choice, and regarded an alternative solution. It can be done either through oral or trans-enterotomy approach and it is used successfully for the localization and treatment of different causes of bleeding ${ }^{29}$.

Late and chronic bleeding occurs 6 weeks and above from time of surgery and mostly is intra-luminal with picture of GIT bleeding, this bleeding mostly occurs with GBP rather than with other types of bariatric operations, and the most common cause is ulcer. Usually we start by conservative management with medical treatment and if no response, endoscopic intervention is the treatment of choice with good results in many centers ${ }^{30}$.

Conclusion: Although the incidence of post bariatric bleeding is decreasing nowadays, it is still a serious problem and we should diagnose it as early as possible. Admission of the patient to the hospital with close observation, full investigations and serial checkup is a must. Surgeon and his team should take proper decision at proper time weather continue conservative treatment or change to surgical 
intervention. Staple line reinforcement surgeons to use reinforcement method to plays an important role in decreasing decrease incidence of post-operative incidence of bleeding, we advise the bleeding.

\section{References}

1- WHO Technical Report Series 894 Obesity: Preventing and Managing The Global Epidemic. World Health Organization; Geneva, Switzerland: 2000.

2- $\quad$ aiswal GR, Jain VK, Dhodapkar SV, Kumathalli KI, Kumar R, Nemawat A, Jain A. J Clin Diagn Res. 2015 Sep;9(9):ZC43-5. doi: 10.7860/JCDR/2015/14663.6489. Epub 2015 Sep 1. PMID:26501011

3- Fajnwaks P, Ramirez A, Martinez P, Arias E, Szomstein S, Rosenthal R (May 2008). "P46: Outcomes of bariatric surgery in patients with BMl less than $35 \mathrm{~kg} / \mathrm{m} 2 "$. Surgery for Obesity and Related Diseases. 4 (3): 329. doi:10.1016/j.soard.2008.03.107.

4- Bergman RN, Stefanovski D, Buchanan TA, Sumner AE, Reynolds, JC, Sebring NG, et € al. A better index of body adiposity. Obesity (Silver Spring). 2011;19(5):1083.

5- Dimitrov DV, Ivanov V, Atanasova M. Advantages of bariatric medicine for individualized prevention and treatments: multidisciplinary approach in body culture and prevention of obesity and diabetes. EPMA J. 2011 Sep;2(3):271-6.

6- Luppino FS, de Wit LM, Bouvy PF, Stijnen T, Cuijpers P, Penninx BW, Zitman FG (March 2010). "Overweight, obesity, and depression: a systematic review and meta-analysis of longitudinal studies". Archives of General Psychiatry. 67 (3): 220-9.

7- Runkel N, Colombo-Benkmann M, Hüttl TP, Tigges H, Mann O, Flade-Kuthe R, Shang E, Susewind M, Wolff S, Wunder R, Wirth A, Winckler K, Weimann A, de Zwaan M, Sauerland S. Evidence-based German guidelines for surgery for obesity. Int J Colorectal Dis. 2011 Apr;26(4):397-404.

8- mehranA.szomstein S.R.Mangement of acute bleeding after laparoscopic Roux-en-Y bypass.obes surg. 2003:13:842-7.

9- Dapri G, Cadiere GB, Himpens J. Reinforcing the staple line duringlaparoscopic sleeve gastrectomy: prospective randomized clinical study comparing three different techniques. Obes Surg. 2010;20:462-7.

10- Gill RS, Whitlock KA, Mohamed R, Sarkhoush K, Birch DW, Karmali S. The role of upper endoscopy in treating postoperative complications in bariatric surgery. J Interv Gastroenterol. 2012;2(1): 37-41.

11- Kehagias I, Zygomalas A, Karavias D, Karamanakos S (2016) Sleeve gastrectomy: Have we finally found the holy grail of bariatric surgery? A review of the literature. Eur Rev Med Pharmacol Sci 20: 4930-4942.

12- Kourosh S, Birch DW, Sharma A, Karmali S (2015) Complications associated with laparoscopic sleeve gastrectomy for morbid obesity: A surgeon's guide. Can J Surg 56: 347-352.

13- Shikora S. The use of staple-line reinforcement during laparoscopic gastric bypass. Obes Surg 2004;14(10)1313-20.

14- Nguyen N, Longoria M, Welbourne, S, et al. Glycolide copolymer staple-line reinforcement reduces staple site bleeding during laparoscopic gastric bypass: a prospective randomized trial. Arch Surg 2005;140(8):773-8.

15- Nguyen NT, Langoria M, Chalifoux S, Wilson SE. Gastrointestinal bleeding after laparoscopic gastric bypass. Obes Surg. 2004;14: 1308-12.

16- Dillemans B, Sakran N, Van Cauwenberge S, Sablon T, Defoort B, Van Dessel E, et al. Standardization of the fully stapled laparoscopic Roux-en-Y gastric bypass for obesity reduces early immediate postoperative morbidity and mortality: a single center study on 2606 patients. Obes Surg. 2009;19:1355-64.

17- Schauer PR, Ikramuddin S, Gourash W, Ramanathan R, Luketich J. Outcomes after laparoscopic roux-en-Y gastric bypass for morbid obesity. Ann Surg. 2000;232:515-29.

18- Bellorin O, Abdemur A, Sucandy I, Szomstein S, Rosenthal RJ. Understanding the signifi cance, reasons, and patterns of abnormal vital signs after gastric bypass for morbid obesity. Obes Surg. 2011;21:707-13.

19- Gentileschi P, Camperchioli I, D'ugo S, et al. Staple-line reinforcement during laparoscopic sleeve gastrectomy using three different techniques: a randomized trial. Surg Endosc. 2012;26(9):2623-9.

20- Shikora SA. The use of staple-line reinforcement during laparoscopic gastric bypass. Obes Surg. 2004;14:1313-20.

21- Silecchia G, Boru CE, Mouriel J, Rossi M, Anselmino M, Morino M, Toppino M, Gaspari A, Gentileschi P, Tacchino R, Basso N. The use of fi brin sealant to prevent major complications following laparoscopic gastric bypass: results of a multicenter, randomized trial. Surg Endosc. 2008;22(11):2492-7.

22- Dapri G, Cadiere GB, Himpens J. Reinforcing the staple line during laparoscopic sleeve gastrectomy: prospective randomized clinical study comparing three different techniques. Obes Surg. 2010;20:462-7.

23- Shikora SA, Mahoney CB. Clinical benefit of gastric staple line reinforcement (SLR) in gastrointestinal surgery: a meta-analysis. Obes Surg. 2015;25:1133-1141.

24- Iqbal M, Manjunath S, Seenath M, Khan A. Massive upper gastrointestinal hemorrhage: an unusual presentation after laparoscopic adjustable gastric banding due to erosion into the celiac axis. Obes Surg. 2008;18:759-60.

25- Strodel WE, Knol JA, Eckhauser FE. Endoscopy of the partitioned stomach. Ann Surg. 1984;200:582-6.

26- Bakhos C, Alkhoury F, Kyriakides T, Reinhold R, Nadzam G. Early postoperative hemorrhage after open and laparoscopic roux-en-y gastric bypass. Obes Surg. 2009;19:153-7.

27- Rabl C, Peeva S, Prado K, James AW, Rogers SJ, Posselt A, et al. Early and late abdominal bleeding after roux-en-y gastric bypass: sources and tailored therapeutic strategies. Obes Surg. 2011;21: 413-20.

28- Strodel WE, Knol JA, Eckhauser FE. Endoscopy of the partitioned stomach. Ann Surg. 1984;200:582-6.

29- Chung RS. Laparoscopy assisted jejunal resection for bleeding leiomyoma. Surg Endosc 1998; 12: 162-3

30- Braley SC, Nguyen NT, Wolfe BM. Late gastrointestinal hemorrhage after gastric bypass. Obes Surg. 2002;12:404-7. 